\title{
A characterization of maximin: corrigendum
}

\author{
Kaname Miyagishima • Kristof Bosmans · Erwin Ooghe
}

Received: 19 March 2014 / Accepted: 22 March 2014 / Published online: 8 April 2014

(C) SAET 2014

Bosmans and Ooghe (2013, p. 155) incorrectly state that the axioms in their theorem are independent. In fact, anonymity is redundant. The theorem may therefore be strengthened as follows.

Theorem A quasi-ordering $R$ on $\mathbb{R}^{n}$ satisfies continuity, weak Pareto and weak Hammond equity if and only if it is maximin.

Proof Let $R$ be a quasi-ordering on $\mathbb{R}^{n}$ that satisfies continuity, weak Pareto and weak Hammond equity. Consider two utility vectors $u, v \in \mathbb{R}^{n}$.

First, we have to show that $u_{(1)}>v_{(1)}$ implies $u P v$. Let $i \in N$ be such that $v_{i}=v_{(1)}$. Construct vectors $w, z \in \mathbb{R}^{n}$ such that

$$
\begin{aligned}
v_{i}<w_{i}<z_{i}< & z_{(2)}=z_{(3)}=\cdots=z_{(n)} \\
& <u_{(1)} \leq \max \left(u_{(n)}, v_{(n)}\right)<w_{(2)}=w_{(3)}=\cdots=w_{(n)} .
\end{aligned}
$$

By weak Pareto, we have $u P z$ and $w P v$. By weak Hammond equity, we have $z R w$. Hence, we obtain $u P v$ using transitivity.

K. Miyagishima $(\varangle)$

JSPS, Waseda University, Totsuka-machi 1-106, Shinjuku-ku, Tokyo, Japan

e-mail: kaname1128@gmail.com

K. Bosmans

Department of Economics, Maastricht University, Tongersestraat 53, 6211 LM Maastricht,

The Netherlands

e-mail: k.bosmans@maastrichtuniversity.nl

E. Ooghe

Center for Economic Studies, Katholieke Universiteit Leuven, Naamsestraat 69, 3000 Leuven, Belgium

e-mail: erwin.ooghe@kuleuven.be 
Second, we have to show that $u_{(1)}=v_{(1)}$ implies $u I v$. This follows from part (ii) of the proof in Bosmans and Ooghe (2013, p. 155).

The standard Hammond equity axiom is stronger than weak Hammond equity (given transitivity). Hence, contrary to what Bosmans and Ooghe (2013, footnote 9) state, Miyagishima's (2010) claim that maximin is the only quasi-ordering satisfying continuity, weak Pareto and Hammond equity is correct.

Acknowledgments Kaname Miyagishima is grateful for the financial support from JSPS KAKENHI (13J06143).

\section{References}

Bosmans, K., Ooghe, E.: A characterization of maximin. Econ. Theory Bull. 1, 151-156 (2013)

Miyagishima, K.: A characterization of the maximin social ordering. Econ. Bull. 30, 1278-1282 (2010) 